\title{
Antibiotikum schützt nicht vor Exazerbationen
}

Miravitlles $M$ et al. Efficacy of moxifloxacin in the treatment of bronchial colonisation

in COPD. Eur Respir J. 2009;34:1066-1071

\section{Hintergrund}

Bis zu $45 \%$ aller Patienten mit mittelbis schwergradiger COPD weisen eine bakterielle Kolonisation mit potenziell pathogenen Mikroorganismen auf. Diese Patientengruppe ist durch gehäufte Exazerbationen gefährdet [1].

Serielle molekulare Typisierungen der bakteriellen Erreger aus dem Sputum von Patienten mit COPD in der stabilen Phase sowie mit akuten Exazerbation weisen darauf hin, dass die kolonisierenden Erreger gehäuft zu akuten Exazerbationen führen.

Die Bedeutung der Kolonisation von Erregern in den Atemwegen für die Entstehung und den Verlauf von akuten Exazerbationen einer COPD ist noch unklar.

\section{Methodik}

Eine spanische Arbeitsgruppe untersuchte in einer randomisierten, kontrollierten Studie 40 chronisch bakteriell besiedelte COPD-Patienten (Durchschnittsalter: 68 Jahre; Einsekundenkapazität $\left[\mathrm{FEV}_{1}\right]$ : $46 \%$ des Sollwerts).

\section{Ergebnisse}

Das Keimspektrum im provozierten Sputum umfasste

- Haemophilus influenzae,

- Haemophilus parainfluenzae,

- Moraxella catarrhalis,

- Pneumokokken,

_Enterobacteriaceae,

- multiple Keime.

Die Patienten erhielten fünf Tage lang entweder Moxifloxacin oder Placebo.

Bei 75\% der mit Moxifloxacin und 30\% der mit Placebo behandelten Patienten verschwanden die Erreger nach zwei Wochen. Nach acht Wochen fanden sich in der Placebogruppe zwar noch immer mehr Erreger, jedoch war der Unterschied statistisch nicht signifikant.
Allerdings kam es zu einer Besiedelung mit neuen Erregern, die in beiden Gruppen ähnlich häufig war $(60 \%$ unter Placebo vs. $70 \%$ unter Moxifloxacin; p > 0,25) (Abb. 1). Der Nachweis einer Rekolonisierung mit neuen potenziell pathogenen Bakterien war mit einem erhöhten Exazerbationsrisiko bei den betroffenen Patienten assoziiert (Odds Ratio $=9,63)$.

\section{Kommentar}

\section{Priv.-Doz. Dr. med. Martin Kohlhäufl, Stuttgart-Gerlingen}

Die Vorstellung, dass die bakterielle Flora bei COPD-Patienten stabil sei, ist naiv. Es kommt permanent zur Eradikation von Erregern, jedoch auch zu einer raschen Neubesiedlung mit neuen pathogenen Erregern. Einzeltherapien zur Erregereradikaeine Therapiealternative darstellen sollen, dann müssen diese in regelmäßigen $A b$ ständen, z.B. alle acht Wochen, gegeben werden. Das ist aus Resistenzgründen jedoch kaum zu verantworten.

Die untersuchte Studienpopulation ist sicher zu klein, um aus den Ergebnissen der Arbeit wesentliche Schlüsse ziehen zu können. Außerdem wird H. parainfluenzae als atemwegspathogen gewertet. Dies ist unter Mikrobiologen umstritten.

Die Auswahl eines Fluorchinolons bei stabilen ambulanten COPD-Patienten ist vor dem Hintergrund der Resistenzproblematik kritisch zu sehen. Moxifloxacin wird nach den Leitlinien aus dem Jahr 2009 nur für die stationäre Therapie mittelschwerer und schwergradiger akuter Exazerbationen der COPD als Alternative (bei Therapieversagen oder Unverträglichkeit) zu Aminopenicillinen (mit Betalactamaseinhibitor) empfohlen [2].

\section{Literatur}

1. Patel IS et al. Relationship between bacterial colonisation and the frequency, character, and severity of COPD exacerbations. Thorax 2002;57:759764

2. Höffken $\mathrm{G}$ et al. Epidemiologie, Diagnostik, antimikrobielle Therapie und Management von erwachsenen Patienten mit ambulant erworbenen tiefen Atemwegsinfektionen sowie ambulant erworbener Pneumonie. 2009 (www.uni-duesseldorf.de/AWMF/II/o82-001.htm) tion sind daher sinnlos. Wenn Antibiotika

\section{Potenziell pathogene Erreger im Sputum}

Abbildung 1

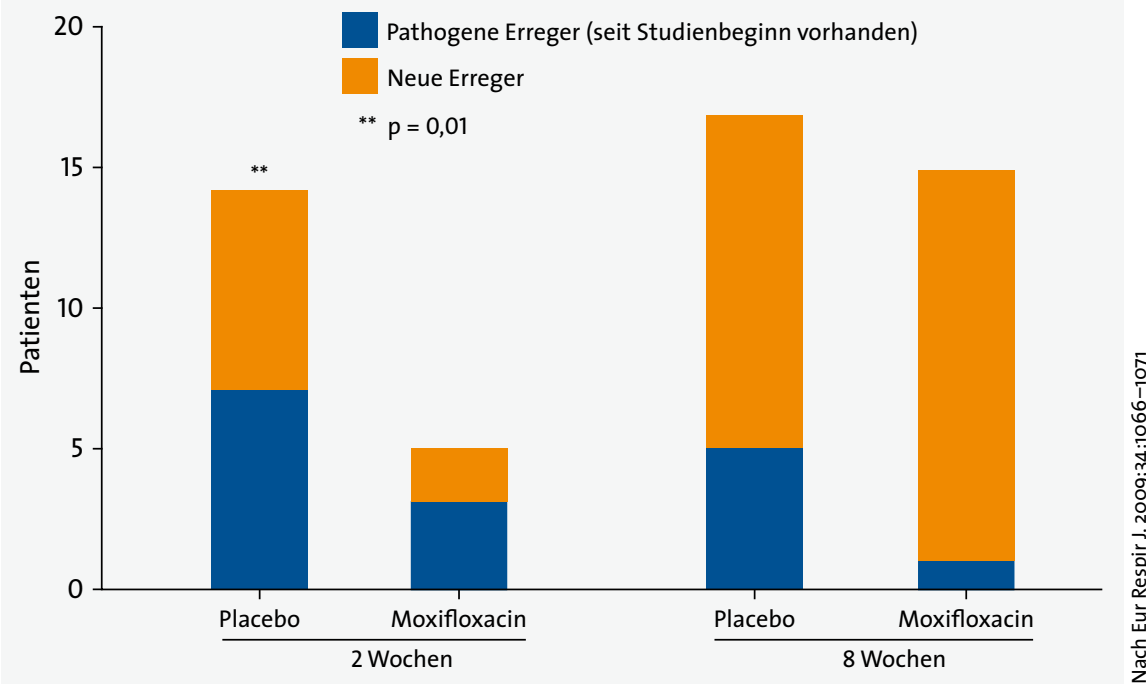

